\title{
Medical Image Segmentation based on Fully Convolutional Network and Minimizing Energy Between Curves
}

\author{
Vo Thi Hong Tuyet ${ }^{1}$, Nguyen Thanh Binh ${ }^{2,3}$ \\ ${ }^{1}$ Faculty of Information Technology, Ho Chi Minh City Open University, Viet Nam \\ ${ }^{2}$ Faculty of Computer Science and Engineering, Ho Chi Minh City University of Technology, VNU-HCM, \\ 268 Ly Thuong Kiet Str, Dist. 10, Ho Chi Minh City, Viet Nam \\ ${ }^{3}$ Vietnam National University Ho Chi Minh City, Linh Trung Ward, Thu Duc District, \\ Ho Chi Minh City, Viet Nam
}

\begin{abstract}
Energy between curves of image has useful for object contour. The edge map is an important task for recognition. The shape that is found by linking between edges will clearly present the useful information of objects. The aim of medical image segmentation is the representation of a medical image into small pieces. In this process, feature extraction must adapt with edge map completely. This paper proposed a solution for medical image segmentation based on fully convolutional network with gradient vector flow snake in bandelet domain. Our approach depends on decomposition in bandelet domain and reconstruction in contour detection by fully convolutional network combining with gradient vector flow snake. To improve the accuracy of the feature's extraction processing, the proposed method detected the edge map in bandelet domain by using fully convolutional network. And its reconstructed objects contour by using gradient vector flow snake combined with the boundary condition. The results of the proposed method have the segmentation clearly with small details of medical images in high-quality and low-quality cases.
\end{abstract}

DOI: $10.18421 /$ TEM94-05

https://doi.org/10.18421/TEM94-05

Corresponding author: Vo Thi Hong Tuyet,

Faculty of Information Technology, Ho Chi Minh City Open University, Viet Nam.

Email: tuyet.vth@ou.edu.vn

Received: 01 July 2020.

Revised: 04 September 2020.

Accepted: 11 September 2020.

Published: 27 November 2020.

(cc) BY-NC-ND (C) 2020 Vo Thi Hong Tuyet \& Nguyen Thanh Binh; published by UIKTEN. This work is licensed under the Creative Commons Attribution-NonCommercialNoDerivs 4.0 License.

The article is published with Open Access at www.temjournal.com
Keywords - medical image segmentation, fully convolutional network, bandelet transform, gradient vector flow snake.

\section{Introduction}

In the state-of-the-art life, object detection is giving a wide range of advantages for community such as: transportation, security, supervision, etc. Any action of object which is concerned in viewport is followed by detection. In this field, the recognition is one of the important tasks for intelligent system. The recognition depends on strong pixels, edges map, contour, segmentation technique, etc. On the other hand, the shape of the object is based on boundary and strong area.

Image segmentation is the useful approach for treatment in medical profession. The result of segmentation shows a wide range of vital information of patients, such as: enlarged or pinched. In simple terms, the difference of colour intensity or the break in boundaries can be a solid base for the status of disease. Therefore, these specialties become a pressing issue in medicine. Daniel [5] and Dilpreet [9] gave a wide range of generations of image segmentation. In this review, there are three of these generations of them, which were mentioned. The first generation includes single or complex thresholds, seed point of region growing, region split/merge, edge tracing for boundary. The second generation includes statistical pattern recognition, clustering, model for contour or surfaces, graph search, neural networks, multiresolution or multiscale methods, geodesic minimal path, target tracking, combination between concepts. The third generation includes atlas-based segmentation, rule-based segmentation, coupled surfaces, shape models, appearance models and deformable organisms. As the above, the algorithm for image segmentation has a wide range of challenges. 
The new generation wavelet transform has many useful for segmentation and object detection, such as: discrete wavelet transforms [1], contourlet for image representation [2], model of nonsubsampled contourlet transform [3], curvelet transform [10] and [11], bandelet transform [7], etc. The scale, threshold [20] or filter [23] are the main idea of the wavelet generation for fusion or segmentation. The connection between multilevel filters or threshold is complex. On the other hand, these solutions face with the lack information of medical images. The denoising is applied for segmentation [8] because of the noise signal created a lot of difficulties for keeping curves. Another reason is that the denoising process has approximation activities. These activities keep strong pixel in all scales and give coefficients for reversion.

The models and texture for segmentation were presented by Gaussian mixture models [4]. Then, the development of this model in bandelet for pancreas segmentation is proposed in [22]. In these ways, Gaussian mixture models applied in texture features or transform for classification and medical image segmentation. Active contour model was also used for development of the object boundaries. The diffusion flow active contours were proposed in [6]. Then, the active contour model was updated, such as: self-affine for energy in [13] and [28], snakes and curvelet transform [16], snakes and contourlet [17], etc.

Multidirectional and multiresolution were the idea of gradient vector flow snakes in [12], [14], [15] and hybrid gradient vector flow in [21]. In addition, the occurring of neural network: convolutional neural network [18], fully convolutional networks [24], convolution [25], manufacturing using convolutional neural network [26] and deep learning [19] are the powerful tools for features representation. Fully convolutional network had applied to segment image and no relationship with other information form scale or flow. The result of network is prediction and it rarely gives better results than domain. While edge map or boundary detection was implemented successfully in the previous phase. Marmanis [27] was proposed boundary detection for upturn semantic image segmentation. Their deeper purpose is classification.

In this paper, we proposed the new method for medical image segmentation by combining fully convolutional network (FCN) with gradient vector flow snake in bandelet domain. Our method has two periods: decomposition and reconstruction. Firstly, we decomposed medical image to predict which pixels belong to the objects. To improve the accuracy of the feature extraction, we detected the edge map in bandelet domain by using fully convolutional network. Secondly, we reconstructed object contour by using gradient vector flow snake combined with the boundary object condition.
Main contribution of this paper is proposal of a method for medical image segmentation in bandelet domain. Specifically, (i) detecting potential candidate pixels which can belong to the object based on fully convolutional network in bandelet domain, (ii) the adapter combined bandelet coefficients with gradient vector flow snakes (GVFS) for segmentation. The rest of this paper is organized as follows: section 2 is the basic knowledge about bandelet transform. Then, we describe the proposed method for medical image segmentation in section 3. Section 4 presented results and experiment clearly. Conclusion and feature work of this paper are shown in the final section.

\section{Bandelet Domain}

The bandelet is similar with other generation wavelet transform about the processing. In [7], a geometric flow of vectors is the basic concept of bandelet domain. However, the edge map that is created by these vectors has special features. Features extraction of this domain is $2^{\mathrm{j}}$ and wavelet coefficients [8] can be defined by equation (1):

where,

$$
\begin{aligned}
& <\mathrm{f}, \psi_{\mathrm{j}} \mathrm{n}>=\mathrm{f}^{*} \psi_{\mathrm{j}}\left(2^{\mathrm{j} *} \mathrm{n}\right) \\
& \psi_{\mathrm{j}}(\mathrm{x})=\frac{1}{2^{j}} \psi\left(-2^{-j} x\right)
\end{aligned}
$$

In the above equation, the form of bandelet function (range in $[0,1]^{2}$ ) has coefficients. The symbol $\psi_{v}[\mathrm{n}]$ is these coefficient of each scale. The equation (3) is the form of bandelets:

$$
b_{v}(x)=\sum_{n} \psi_{v}[n] \psi_{j n}(x)
$$

The processing of bandelets includes two periods: decomposition and reconstruction. In the first period, the reshape vector is created by space domain. This result comes from the quad-tree model for each scale. Each vector had given the feature of each level. In the simple term, features extraction can be applied in this. The quad-tree model can be defined by recursion process of dividing from the other step. An example for this model is that each input image divides four parts with the same size. Then, each part had divided four sub-parts and is continuous with others. The stop condition of this recursion is based on the depth of decomposition. In other ways for decomposition, the concept of low-pass and highpass were presented. Low-pass was continued for this dividing, but high-pass was not. The second period, the reconstruction is done by the reverse process with the previous periods. The summary of this step is calculating from wavelet coefficients. If a solution uses deep learning or neural network for features extraction, the predicted value is the primary key for the detection of classification or the reconstructed level. 


\section{The Proposed Method for Medical Image Segmentation in Bandelet Domain}

The energy between pixels is useful for object detection. The boundary of objects is affected by this energy. The concept of minimizing energy is handled in active contour model - Snakes. In this research, we propose gradient vector flow snake in features, which were extracted from fully convolutional network. Figure 1. presents our proposed method clearly. The proposed method includes two periods: Firstly, we decompose medical image into multi reshape vector (reshape vector 1 , reshape vector $2, \ldots$, reshape vector $\mathrm{n}$ ). Then, each of them will be applied the fully convolutional network for feature extraction. Secondly, we reconstructed objects contour by using gradient vector flow snake combined with the boundary object condition. The result of each reshape vector will be network of convolutional. The computation model for this step is as the below Figure 2. In each reshape vector, the number of pixels is the same size of the input image of model.

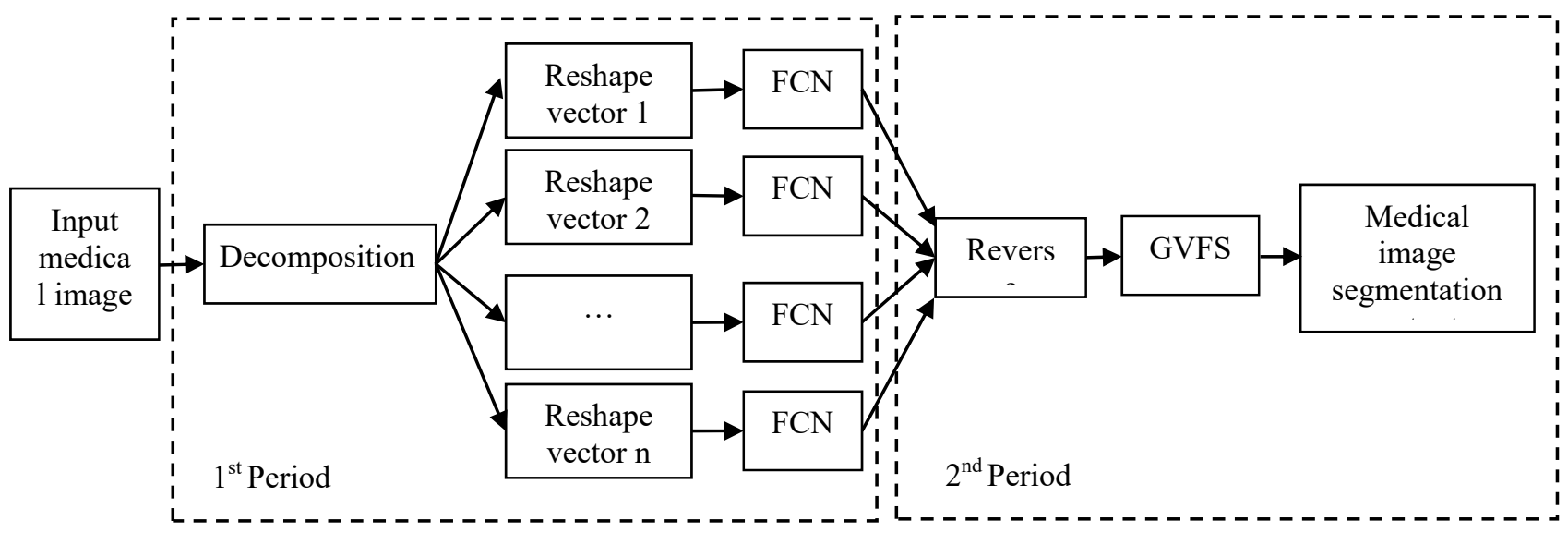

Figure 1. The processing of the proposed method for medical image segmentation in bandelet domain.

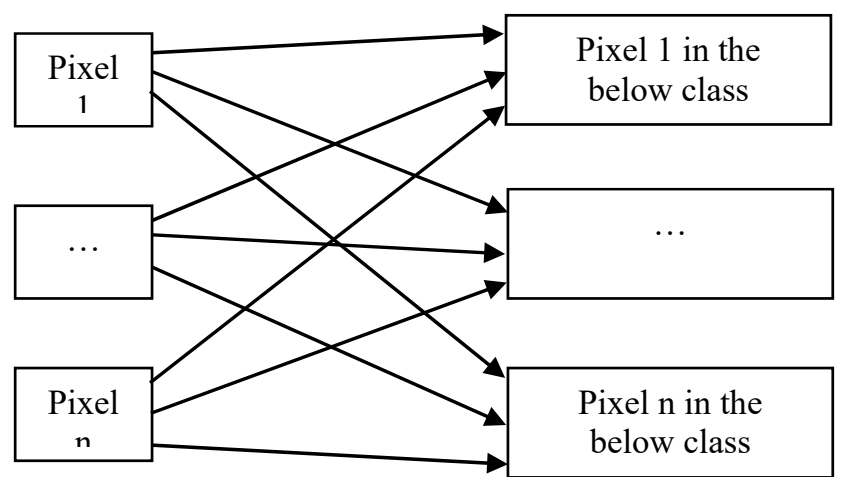

Figure 2. The computation model of the fully convolutional network in the proposed method

\subsection{Decomposition Image to Predict which Pixels Belong to the Objects in Bandelet Domain.}

The convolutional network was presented in [24]. FCN can be called a representative of semantic segmentation. The primary key of semantic segmentation is the differences between semantics and location. FCN is called the translation invariance. Here, the size of each layer in nets is three-dimensional array of spatial and the number of channel dimension. The proposed method uses the same size with the above layer size. However, in the final layer, we use 1000 pixels for sigmoid prediction.
We defined the size $(\mathrm{H} \times \mathrm{W} \times \mathrm{D})$ of the input image, where $\mathrm{H}$ and $\mathrm{W}$ are the value of spatial dimensions, $\mathrm{D}$ is the size of channels dimensions.

In the first layer, the size $(\mathrm{H} \times \mathrm{W} \times \mathrm{D})$ of the input image calculated the pooling value for the previous layer. The higher layer will correspond with position of image by path-connected receptive fields. The next layers can be described by equation (3):

$$
y(i, j)=f_{k s}\left(\left\{x_{s i}+\delta_{i, s j}+\delta_{j}\right\} 0 \leq \delta_{i}, \delta_{j} \leq k\right)
$$

where, $\mathrm{k}$ is the kernel size, $\mathrm{s}$ is the number of factors, $f_{k s}$ is the matrix or spatial which depends on the type of the layer.

After the decomposition, the reshape vector of any scale also has the features of objects. The proposed method applied convolution network in each of them. The duplication between pixels together will be removed by linear structures when a medical image is decomposed. Convolution, average pooling, max pooling or activation are calculated by $f_{k s}$. The final step of FCN is predicted by soft max equation (5):

$$
S=\frac{\exp \left(z_{i}\right)}{\sum_{j=1}^{C} \exp \left(z_{j}\right)}
$$

where, $z_{i}$ is the pixel $i^{\text {th }}$ which joins into networks. From the probability value of prediction $(0-1)$, we decided the segmentation results. Then, the synthesis is calculated as in Figure 3. 


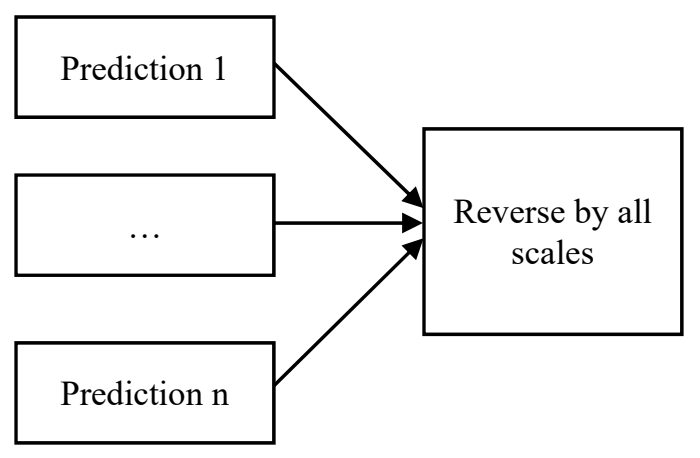

Figure 3. The process from fully convolutional network to inversion

The bandelet coefficients have an effect in the previous step and do not have it in the present. This value is not applied because the conv and pool class of FCN give the upsampled prediction in linear regions. All the below steps are only based on scales of decomposition to build the output images.

\subsection{Reconstruction Objects Contour in Image Depend on Boundary Object Condition}

Derived from the minimizing energy activities in boundaries of objects, active contour model (snakes) includes five steps:

(i) Firstly, the edge map was established by the prediction of the previous period. Another name of this map could be called the self-affine maps. These parameters for edge detection gave energy between edge for minimizing process. The edge map has three benefits: the gradient, the vicinity and the homogeneity. The first benefit was influenced by a wide range of intensity of the input pixels. The second and third benefit were applied for constructing of region objects.

(ii) Secondly, the minimum cost. The cost was defined by forces in each scale and intensity of the original domain. The internal energy of spline due to bending $\left(E_{\text {int }}\right)$ is presented as equation (6).

$$
E_{\text {int }}=\left(\alpha(s)\left|v_{s}(s)\right|^{2}+\beta(s)\left|v_{s}(s)\right|^{2}\right) / 2
$$

where, $\alpha(s)$ is a user-defined weights. $\beta(s)$ is for the smoothing term. $\mathrm{v}(\mathrm{s})=(\mathrm{x}(\mathrm{s}), \mathrm{y}(\mathrm{s}))$ is the position of a snake parametrically.

(iii) Thirdly, we setup the connection for the forces together. The linking can be defined by the rise of the external constraint forces $\left(\mathrm{E}_{\mathrm{con}}\right)$ presented as equation (7).

$$
E_{\text {con }}=\frac{1}{2}\left(\alpha(s)\left|v_{s}(s)\right|^{2}\right)
$$

(iv) Fourthly, the sum value of the internal energy of spline due to bending, the image forces, and the external constraint forces presented as equation (8):

$$
E_{\text {image }}=w_{\text {line }} E_{\text {line }}+w_{\text {edge }} E_{\text {edge }}+w_{\text {term }} E_{\text {term }}
$$

where, $\mathrm{w}_{\text {line }}, \mathrm{w}_{\text {edge }}$ and $\mathrm{w}_{\text {term }}$ are weights of these salient features.

(v) GVFS is the total of smoothness and edge energy. It can be simplified as:

$$
E(u, v)=\iint \mu|\nabla V|^{2} d x d y+\iint|V-\nabla f|^{2} d x d y
$$

where, $\mu$ is a positive weight to control the scale between smoothness and edge energy, $(\mathrm{x}, \mathrm{y})$ is the dynamic scheme, $\nabla^{2}$ is the Laplacian operator: $\mathrm{E}(\mathrm{u}$, v) must satisfy the Euler-Lagrange:

$$
\mu \nabla^{2} V-(V-\nabla f)\left(f_{x}^{2}+f_{y}^{2}\right)=0
$$

The reconstruction is done by imageapproximation process, the minimize of viation can be called (f). The bandelet coefficients (f) was calculated for the region geometric element as low. Obtained smooth flow features and directions are presented as equation (11):

$$
R[u]=\sum_{n=1}^{2^{k-1}} \alpha_{n} B\left(2^{-1} u-n\right)
$$

where, $\alpha$ is the spline expansion coefficients of scale; $u$ is the spline index. Equation (11) explains that these flows can be restored by controlling the spline. On the other hand, the values of equation (7), equation (8) and equation (9) were effectuated for minimizing. The improvement of the solution of this research includes two points based on the bandelet coefficients:

- The prediction replanting,

- According to equation (11), implement practiced replanting segmentation method.

\section{Experiments and Results}

Medical image segmentation requires high accuracy and avoids loss of information in the images. This section will show the results for medical image segmentation by the proposed method and compare it with the other methods.

\subsection{Materials and Dataset}

Experiments are developed in Matlab R2018a language and carried out on computer of Intel core i7, 3.4GHZ CPU, 16 GB DDR3 memory. Our dataset is the medical images including many types (lung, bone, etc). Some medical images in this dataset (dataset test) are presented in Figure 4. 


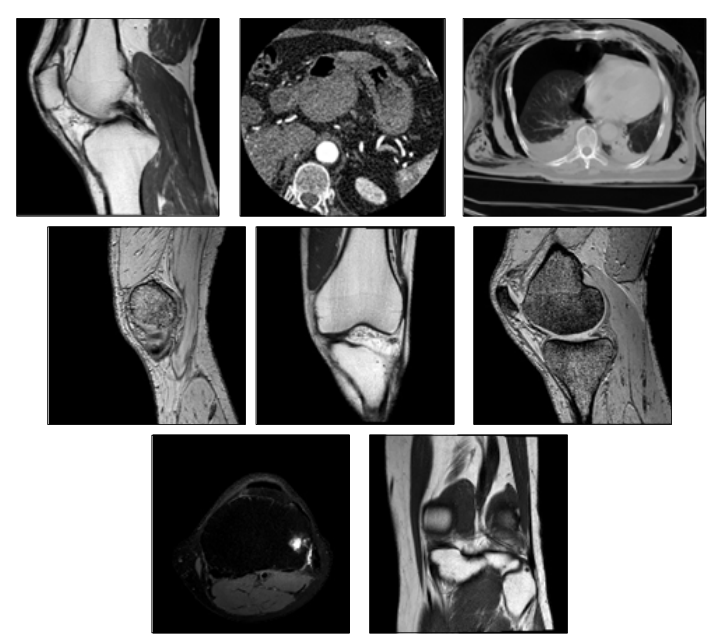

Figure 4. Some medical image in dataset test

This dataset is DICOM images [29]. They include many kinds of medical images as: CT, MR, MRI, etc. These parts of patient's body are presented clearly in them with color or gray. From these collections, an approach exporting these datasets includes 1500 medical images with size: $512 \times 512$ in gray. Each medical image is the storage information of patient's body in each state. Therefore, the quality of medical images is unstable because the export time is not fixed.

\subsection{Evaluation and Experimental Results}

In this sub-section, we test the proposed method with the above dataset. We also compare the results with the other methods such as Zhuo method [21], Evan method [24] and Jordi method [26]. To evaluate the results of these methods, we are not only making comparision by the naked eye but also by the Jaccard index (JI). The JI is presented as equation (11):

$$
J I(A, B)=\frac{|A \cap B|}{|A \cup B|} \times 100
$$

where, A is extracted region and B is true region. The higher the value of JI is, the better it is. JI value is the presentation of the same of testing and ground-truth in dataset. Here, we show some of our results in dataset. Figure 5. shows some results of the proposed method with the other methods.
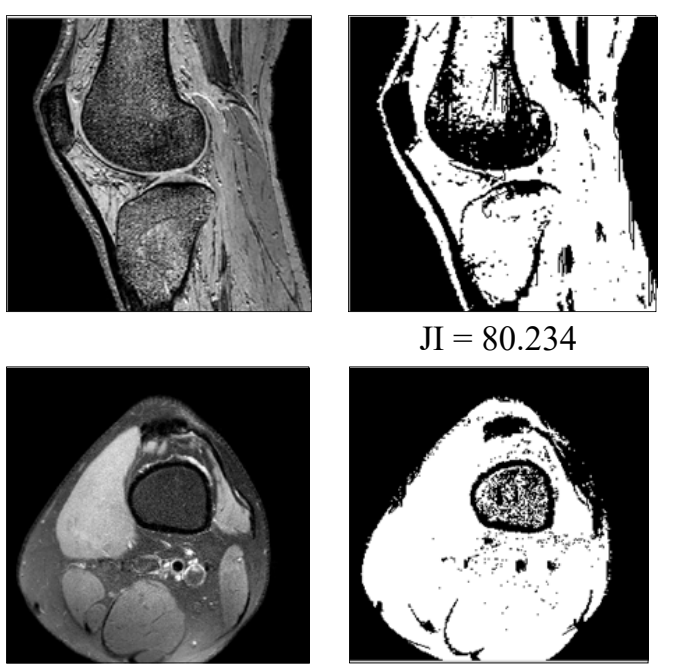

$\mathrm{JI}=80.4097$
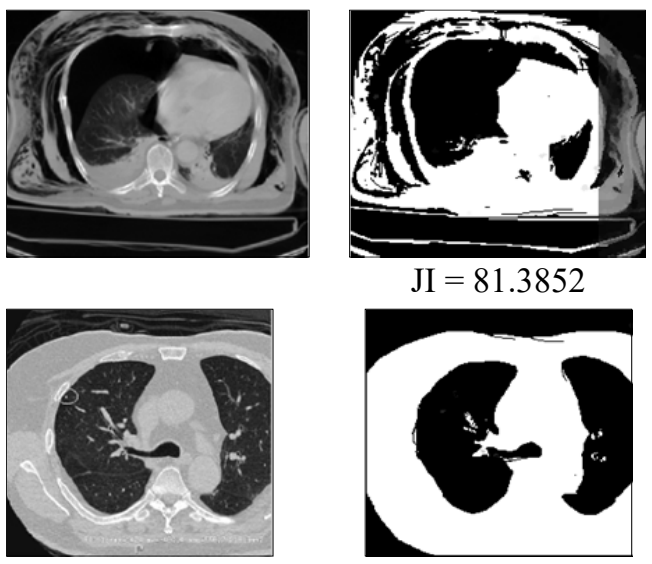

$\mathrm{JI}=81.3852$

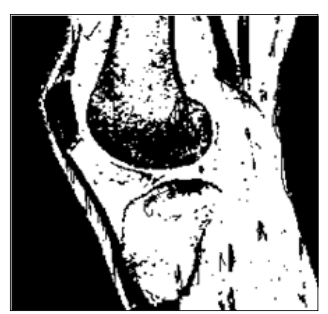

$\mathrm{JI}=80.9556$

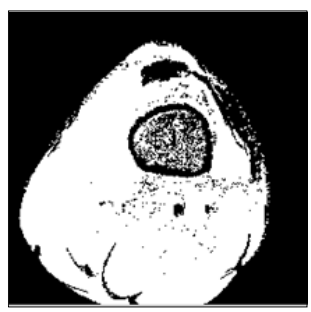

$\mathrm{JI}=81.1129$

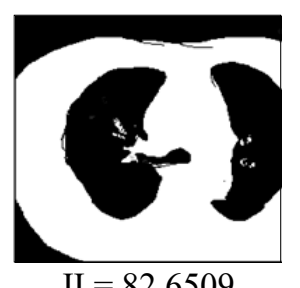

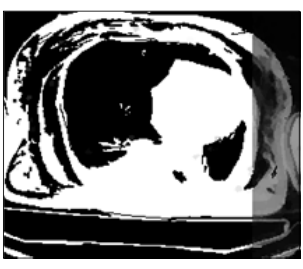

$\mathrm{JI}=81.6923$

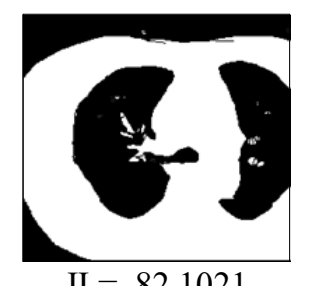

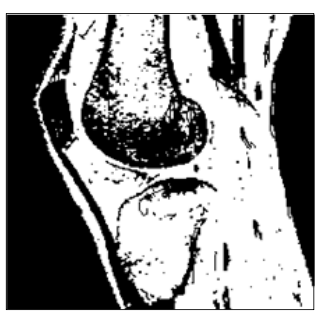

$\mathrm{JI}=81.7614$

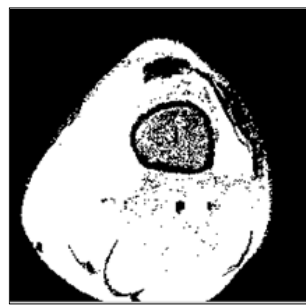

$\mathrm{JI}=81.8289$
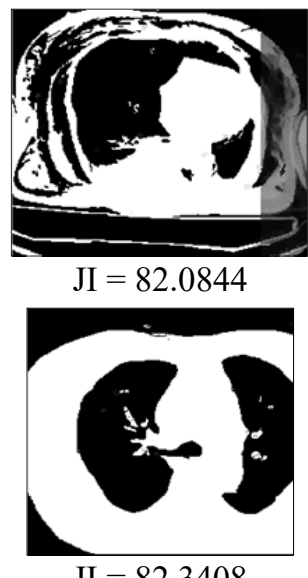

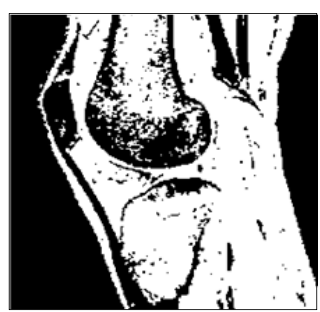

$\mathrm{JI}=86.9915$

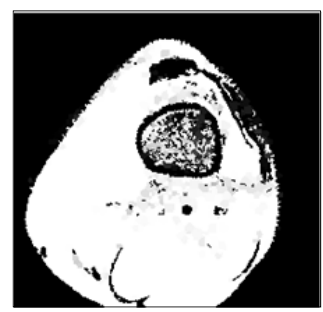

$\mathrm{JI}=87.0289$

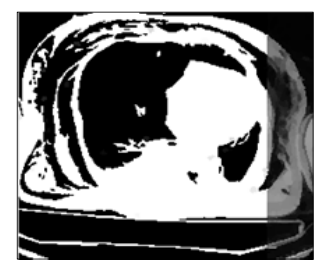

$\mathrm{JI}=87.9844$

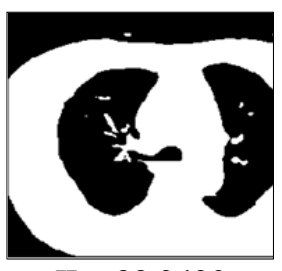

$\mathrm{JI}=88.3429$ 


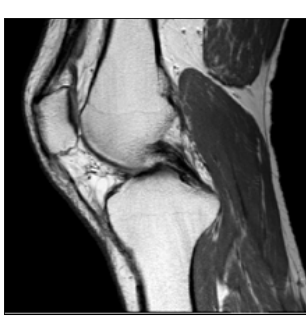

(a)

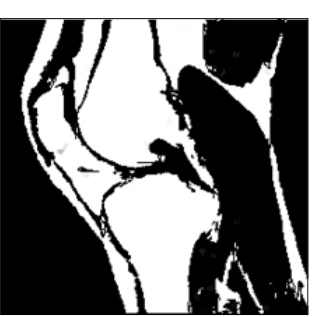

$\mathrm{JI}=83.12$

(b)

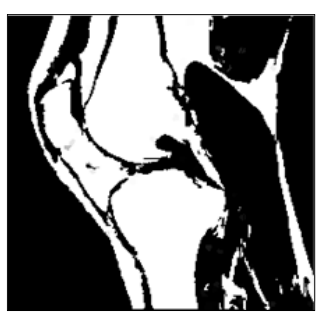

$\mathrm{JI}=83.6189$

(c)

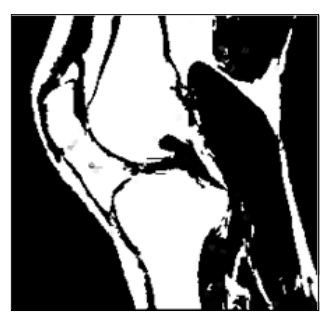

$\mathrm{JI}=82.111$

(d)

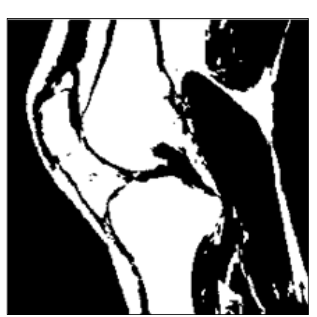

$\mathrm{JI}=89.2111$

(e)

Figure 5. The results of segmentation by the proposed methods and the other methods.
(a). Medical images original
(b). The results of Zhuo method [21]
(c). The results of Evan method [24]
(d). The results of Jordi method [26]
(e). The results of the proposed method

In Figure 5., Figure 5(a) is the image's original in our dataset. Figure 5(b) is the result of snake model based on hybrid gradient vector flow (Zhuo method [21]). Figure 5(c) is the results of fully convolutional network (Evan method [24]). Figure 5(d) is the results of improving pixel-wise semantic segmentation and convolutional neural network (Jordi method [26]). And Figure 5(e) is the result of the proposed method. By visualization, we see that the proposed method results are better than the other results methods. For objective evaluation, we use quantitative methods to compare the results of the above methods. As the above dataset, our dataset has 1500 medical images. The average JI value of all medical images is presented in Table 1 .

Table 1. The average JI value of the above methods

\begin{tabular}{||l|c||}
\hline \multicolumn{1}{|c|}{ The methods } & $\begin{array}{c}\mathbf{1 5 0 0} \\
\text { images } \\
\text { from } \\
\text { dataset }\end{array}$ \\
\hline The average JI value of Zhuo method [21] & 79.5 \\
\hline The average JI value of Evan method [24] & 82.7 \\
\hline The average JI value of Jordi method [26] & 84.4 \\
\hline The average JI value of the proposed method & 89.3 \\
\hline
\end{tabular}

From Table 1., we can see that the result of the proposed method is better than the other methods. The reason of the results of the proposed method is better than the others because the proposed method detected good boundaries. In the proposed method, the contours are improved by the quality of pixels and the edge map clearly. The process for quality of pixels depends on the bandelet coefficients, and the edge map is clear by FCN for calculating. Then, GVFS will give the clear regions of objects.

In Figure 6., we show some results of the comparison with high-quality medical images. The red colour is the results of our approach; the other colours are the results of Zhuo method [21], Evan method [24] and Jordi method [26], respectively. The value of Peak Signal-to-Noise Ratio (PSNR) is used for this valuation. The high-quality of medical images has PSNR from 22.5 to $27 \mathrm{db}$, and the JI values of the other methods are compared together. The vertical axis is the JI values of each method. The PSNR values are presented in horizontal axis. From the data from Figure 6., we can see that the proposed method gives better results than the segmentation result of the others. The others have nearly the same results. However, the ambiguity occurs in $24 \mathrm{db}$ to $26 \mathrm{db}$ of PNSR.

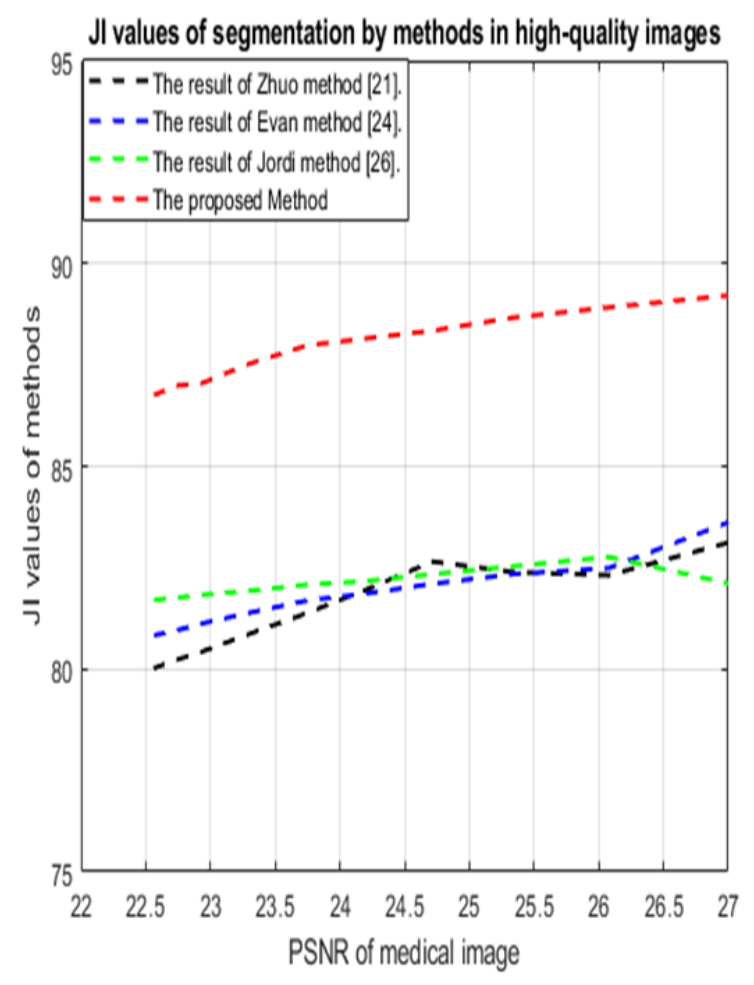

Figure 6. The JI value of the proposed method with the other methods in high-quality medical images

The PSNR value, which is choosen for presenting in here, is a popular value for evaluating the quality of images. The PSNR value from 22.5 to $27 \mathrm{db}$ is the proof of high-quality images. 

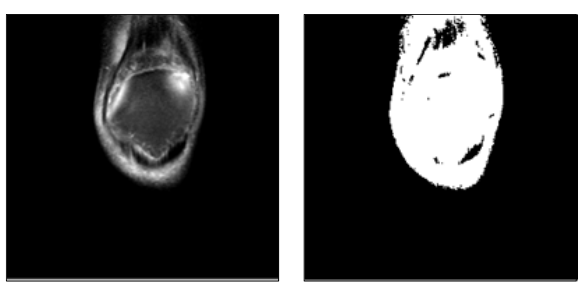

$\mathrm{JI}=70.94$

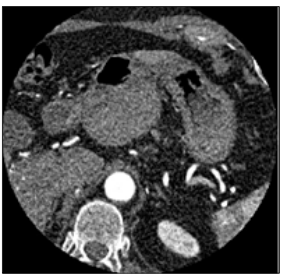

a)

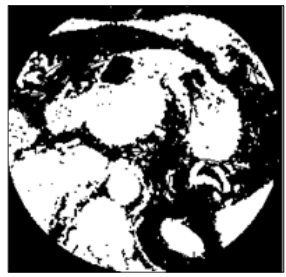

$\mathrm{JI}=76.913$

(b)

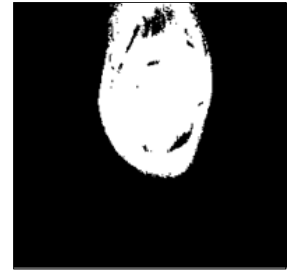

$\mathrm{JI}=71.8346$

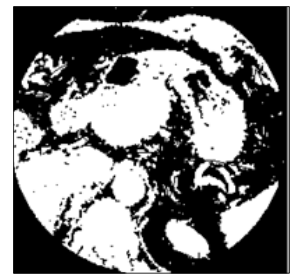

$\mathrm{JI}=75.55$

(c)

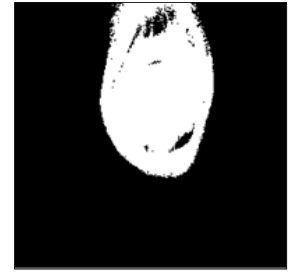

$\mathrm{JI}=71.996$

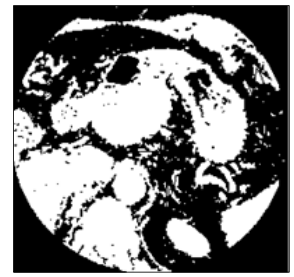

$\mathrm{JI}=75.87$

(d)

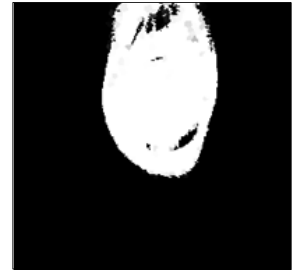

$\mathrm{JI}=72.067$

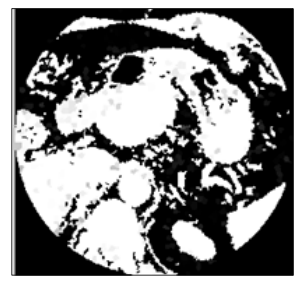

$\mathrm{JI}=75.913$

(e)

Figure 7. The results of segmentation by the proposed methods and the other method in the low-quality medical-image images.

(a). Medical images original

(b). The results of Zhuo method [21]

(c). The results of Evan method [24]

(d). The results of Jordi method [26]

(e). The results of the proposed method

On the other hand, Figure 7. also shows results for some original images and the results of the proposed method with the other methods in low-quality medical image cases. In Figure 7., the proposed method also gives the results better than other methods approach by JI values in the low-quality medical images case. Figure $7(a)$ is the normal medical image, which has low-quality. Figure 7(b), 7(c), Figure 7(d) and Figure 7(e) are the result of Zhuo method [21], Evan method [24], Jordi method [26] and the proposed method, respectively. In all of Figure 7., the JI value of the proposed method is the highest. On the other hand, we also test the results of proposed method in low-quality medical images, which have gaussian noise and blur. The comparison also practices with 1500 medical images of the above dataset. Figure 8. is the chart which compares the results in low-quality medical image case between our approach with the others methods.

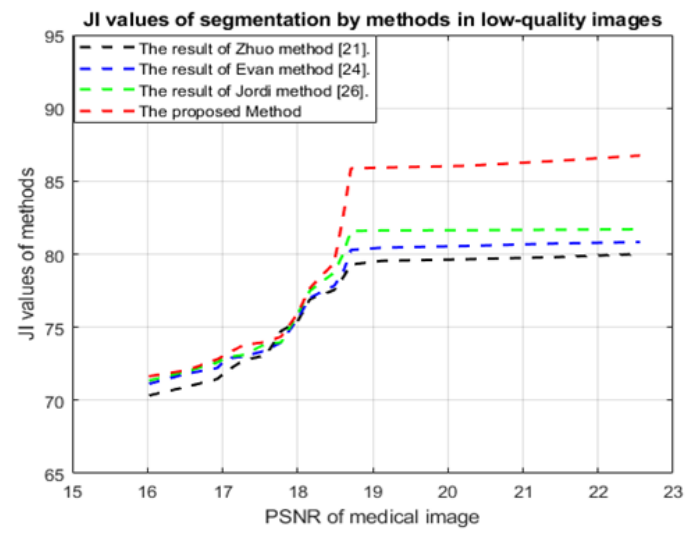

Figure 8. The JI value results of the proposed method with the other methods in low-quality medical images
In Figure 8., the vertical axis is the value of JI of ways and the horizontal axis is the PSNR of medical image for input processing. From the above data, low-quality medical images which have PSNR value from $16 \mathrm{db}$ to $18 \mathrm{db}$ has JI values have not big change between four methods. However, from $18.5 \mathrm{db}$ to more, the JI of our method gives higher than the others.

Therefore, we can infer that the proposed method not only fits with medical segmentation in highquality but also in low-quality. The proposed method gives the better results with the other methods because of the clear featured. In each scale of bandelet domain, we combined the synthesis of prediction and the size retention for showing object regions. The utilization of bandelet transform is also useful for information of medical images. The another reason for good results is snakes includes: smoothing and continuity of the curve of each object in image; the development of outside energy based on the curve growths. The inversion of bandelet transform and FCN has only the prognostication about regions of pixels. We had applied GVFS overcomes drawbacks of the traditional snake model:

- The depending on seed point for the initial step.

- Confusion of vertices.

\section{Conclusion and Feature Works}

With medical images, the contour or regions of object is very important to support the doctor in treatment. However, noise or blur in storage process gives a wide range of difficulties for object detection 
and segmentation. In this paper, we proposed a new method for medical segmentation combined fully convolutional network with gradient vector flow snakes in bandelet domain. The decomposition of this domain is the environment for FCN acting. The output of the previous step is the input for the next step is gradient vector flow snakes. Energy is the helpful between boundaries of object. The quality of images will influence it more or less. The proposed method uses GVFS for segmentation for minimizing energy between curves in image. The results of the proposed method are better than the other recent solutions by comparison the Jaccard index values. This comparison result is not only in case of the high quality but also in the low-quality medical images.

In the future work, deep learning or neural network will be applied clearly in segmentation process. The kind of deep learning is extremely useful for feature extraction of objects. We will expand by the size and the number of layers that help to keep information of input images.

\section{Acknowledges}

This research is funded by Ho Chi Minh City Open University under grant number E2018.07.2

\section{References}

[1]. Kociołek, M., Materka, A., Strzelecki, M., \& Szczypiński, P. (2001, September). Discrete wavelet transform-derived features for digital image texture analysis. In international conference on signals and electronic systems (pp. 99-104).

[2]. Do, M. N., \& Vetterli, M. (2005). The contourlet transform: an efficient directional multiresolution image representation. IEEE Transactions on image processing, 14(12), 2091-2106.

[3]. Arthur L. da Cunha, Jianping Zhou \& Minh N. Do. (2005). Nonsubsampled Contourlet Transform: Theory, Design, and Applications. IEEE Trans. Img. Proc, 3089-3101.

[4]. Da Cunha, A. L., Zhou, J., \& Do, M. N. (2006). The nonsubsampled contourlet transform: theory, design, and applications. IEEE transactions on image processing, 15(10), 3089-3101.

[5]. Permuter, H., Francos, J., \& Jermyn, I. (2006). A study of Gaussian mixture models of color and texture features for image classification and segmentation. Pattern Recognition, 39(4), 695-706.

[6]. Withey, D. J., \& Koles, Z. J. (2008). A review of medical image segmentation: methods and available software. International Journal
Bioelectromagnetism, 10(3), 125-148.

[7]. Wu, Y., Wang, Y., \& Jia, Y. (2013). Adaptive diffusion flow active contours for image segmentation. Computer Vision and Image Understanding, 117(10), 1421-1435.
[8]. Peyré, G., \& Mallat, S. (2008). Orthogonal bandelet bases for geometric images approximation. Communications on Pure and Applied Mathematics: A Journal Issued by the Courant Institute of Mathematical Sciences, 61(9), 1173-1212.

[9]. Binh, N. T., Tuyet, V. T. H., \& Vinh, P. C. (2013, November). Ultrasound images denoising based context awareness in bandelet domain. In International Conference on Context-Aware Systems and Applications (pp. 115-124). Springer, Cham.

[10]. Kaur, D., \& Kaur, Y. (2014). Various image segmentation techniques: a review. International Journal of Computer Science and Mobile Computing, 3(5), 809-814.

[11]. Shan, H., \& Ma, J. (2010). Curvelet-based geodesic snakes for image segmentation with multiple objects. Pattern Recognition Letters, 31(5), 355-360.

[12]. Hamdi, M. A. (2011). Modified algorithm markercontrolled watershed transform for image segmentation based on curvelet threshold. Canadian Journal on Image Processing and Computer Vision, 2(8), 88-91.

[13]. Zhou, H., Li, X., Schaefer, G., Celebi, M. E., \& Miller, P. (2013). Mean shift based gradient vector flow for image segmentation. Computer Vision and Image Understanding, 117(9), 1004-1016.

[14]. Saadatmand-Tarzjan, M. (2015). Self-affine snake for medical image segmentation. Pattern Recognition Letters, 59, 1-10.

[15]. Zhang, R., Zhu, S., \& Zhou, Q. (2016). A novel gradient vector flow snake model based on convex function for infrared image segmentation. Sensors, 16(10), 1756.

[16]. Zhu, S., \& Gao, R. (2016). A novel generalized gradient vector flow snake model using minimal surface and component-normalized method for medical image segmentation. Biomedical Signal Processing and Control, 26, 1-10.

[17]. Tuyet, V. T. H. (2016, March). Active contour based on curvelet domain in medical images. In International Conference on Nature of Computation and Communication (pp. 325-333). Springer, Cham.

[18]. Nageswara Reddy, P., Mohan Rao, C. P. V. N. J., \& Satyanarayana, C. (2017). Brain $\mathrm{Mr}$ Image Segmentation By Modified Active Contours And Contourlet Transform. ICTACT Journal on Image \& Video Processing, 8(2).

[19]. Vardhana, M., Arunkumar, N., Lasrado, S., Abdulhay, E., \& Ramirez-Gonzalez, G. (2018). Convolutional neural network for bio-medical image segmentation with hardware acceleration. Cognitive Systems Research, 50, 10-14.

[20]. Roth, H. R., Shen, C., Oda, H., Oda, M., Hayashi, Y., Misawa, K., \& Mori, K. (2018). Deep learning and its application to medical image segmentation. Medical Imaging Technology,36(2), 63-71.

[21]. Hagargi, P. A., \& Shubhangi, D. (2018). Brain tumor MR image fusion using most dominant features extraction from wavelet and curvelet transforms. Brain, 5(05), 33-38. 
[22]. Yu, Z., Wang, Q., Xiong, W., Zhang, C., \& Hu, H. (2018). Segmentation of cardiac tagged MR images using a snake model based on hybrid gradient vector flow. Multimedia Tools and Applications, 77(17), 21879-21904.

[23]. Nguyen Huu The, Vo Thi Hong Tuyet, Nguyen Mong Hien \& Nguyen Thanh Binh. (2019). Pancreas Segmentation in Medical Images Based on Gaussian Mixture Model in Bandelet Domain. 7th International Conference on the Development of Biomedical Engineering in Vietnam (BME7), 493-497.

[24]. Zhang, J., Xiu, X., Zhou, J., Zhao, K., Tian, Z., \& Cheng, Y. (2020). A novel despeckling method for medical ultrasound images based on the nonsubsampled shearlet and guided filter. Circuits, Systems, and Signal Processing, 39(3), 1449-1470.

[25]. Shelhamer, E., Long, J., \& Darrell, T. (2017). Fully convolutional networks for semantic segmentation. IEEE transactions on pattern analysis and machine intelligence, 39(4), 640-651.
[26]. Wang, P., Chen, P., Yuan, Y., Liu, D., Huang, Z., Hou, X., \& Cottrell, G. (2018, March). Understanding convolution for semantic segmentation. In 2018 IEEE winter conference on applications of computer vision (WACV) (pp. 1451-1460). IEEE.

[27]. Minnema, J., van Eijnatten, M., Kouw, W., Diblen, F., Mendrik, A., \& Wolff, J. (2018). CT image segmentation of bone for medical additive manufacturing using a convolutional neural network. Computers in biology and medicine, 103, 130-139.

[28]. Marmanis, D., Schindler, K., Wegner, J. D., Galliani, S., Datcu, M., \& Stilla, U. (2018). Classification with an edge: Improving semantic image segmentation with boundary detection. ISPRS Journal of Photogrammetry and Remote Sensing, 135, 158-172.

[29]. Yang, X., \& Jiang, X. (2020). A hybrid active contour model based on new edge-stop functions for image segmentation. International Journal of Ambient Computing and Intelligence (IJACI), 11(1), 87-98. 